\title{
Becoming Consistent: Developmental Reductions in Intraindividual Variability in Reaction Time Are Related to White Matter Integrity
}

\author{
Christian K. Tamnes, Anders M. Fjell, Lars T. Westlye, Ylva Østby, and Kristine B. Walhovd \\ Center for the Study of Human Cognition, Department of Psychology, University of Oslo, 0317 Oslo, Norway
}

Cognitive development is known to involve improvements in accuracy, capacity, and processing speed. Less is known about the role of performance consistency, and there has been virtually no empirical examination of the neural underpinnings of within-person variability in development. In a sample of 92 healthy children and adolescents aged 8-19 years, we aimed to characterize age-related changes in trial-to-trial intraindividual variability (IIV) of reaction time (RT) and to test whether IIV is related to white matter (WM) integrity as indexed by diffusion tensor imaging. IIV was quantified as the SD of correct RTs in a speeded arrow flanker task, and Tract-Based Spatial Statistics was used to test relationships with diffusion characteristics. Large age-related reductions in IIV in both simple congruent trials and more complex incongruent trials were found. Independently of sex, age, and median RT (mRT), lower IIV was associated with higher fractional anisotropy and lower overall diffusivity. Effects were seen for IIV in one or both trial types in the corticospinal tract, the left superior longitudinal fasciculus, the uncinate fasciculus, the forceps minor, and in the genu and splenium of the corpus callosum. There were no significant associations between $\mathrm{mRT}$ and any of the diffusion indices. The findings support the proposition that developmental reductions in IIV reflect maturation of WM connectivity and highlight the importance of considering within-person variability in theories of cognitive development and its neurobiological foundation.

\section{Introduction}

Investigations of the neural mechanisms underlying behavioral change are fundamental to the understanding of cognitive development in childhood and adolescence. Cognitive development is typically described in terms of improvements in accuracy, capacity, or processing speed and is operationalized as mean level of performance across multiple measurements (e.g., Waber et al., 2007). Less is known about the role of within-person variability in development. Within persons, variability has been operationalized in two ways: across tasks, referred to as intraindividual differences or dispersion, and across trials or sessions of the same task, referred to as intraindividual variability (IIV) or inconsistency (Hultsch et al., 2002; Li et al., 2004a; MacDonald et al., 2009).

The preponderance of data on within-person variability concerns IIV of reaction time (RT) in older adults, with studies showing age-related increases in performance variability and concomitant impairments in various cognitive functions (Lindenberger and von Oertzen, 2006; MacDonald et al.,

Received Sept. 19, 2011; revised Nov. 16, 2011; accepted Nov. 29, 2011.

Author contributions: C.K.T., A.M.F., and K.B.W. designed research; C.K.T. and Y. Ø. performed research; C.K.T., A.M.F., and L.T.W. analyzed data; C.K.T., A.M.F., L.T.W., Y. Ø., and K.B.W. wrote the paper.

This work was supported by grants from the Norwegian Research Council (177404/W50, 186092/V50 to K.B.W., and 204966 to L.T.W.), the University of Oslo (K.B.W.), and the Department of Psychology, University of Oslo (A.M.F. and C.K.T.).

The authors declare no competing financial interests.

Correspondence should be addressed to Christian K. Tamnes, Department of Psychology, University of Oslo, P.O. Box 1094 Blindern, 0317 0slo, Norway. E-mail: c.k.tamnes@psykologi.uio.no.

DOI:10.1523/JNEUROSCI.4779-11.2012

Copyright $\odot 2012$ the authors $\quad 0270-6474 / 12 / 320972-11 \$ 15.00 / 0$
2009). IIV across the life span is characterized by a U-shaped function, such that childhood and senescence are associated with stronger inconsistency (Li et al., 2009, 2004b; Williams et al., $2005,2007)$. Whereas differences in processing speed have been theorized to underlie many of the cognitive differences between children and adults (Fry and Hale, 1996), studies focused on developmental IIV changes are lacking.

A growing number of studies link IIV to structural brain characteristics in adults, and a key role of white matter (WM) alterations in increased IIV in aging is suggested (Anstey et al., 2007; Bunce et al., 2007; Walhovd and Fjell, 2007; Ullen et al., 2008). Importantly, two recent diffusion tensor imaging (DTI) studies (Fjell et al., 2011; Moy et al., 2011) found relationships between performance variability and the quality of WM and that the strength of these associations increased in older adults. DTI is a neuroimaging technique that is sensitive to the self-diffusion of water molecules and provides quantitative measures of WM microstructural integrity and connectivity (Basser and Jones, 2002; Beaulieu, 2002; Le Bihan, 2003). Various indices can be derived from the estimated diffusion tensor, including fractional anisotropy (FA), which indexes degree of net directionality in diffusion, and mean diffusivity (MD), reflecting average magnitude of diffusion. Additionally, diffusion along [axial diffusivity $(\mathrm{AD})$ ] and across [radial diffusivity (RD)] the main axis of the diffusion tensor can be estimated. Developmental changes in diffusion indices have consistently been reported in the form of FA increases and overall diffusivity decrease, with prolonged maturation of association tracts compared with projection and commissural tracts (Tamnes et al., 2010a; Westlye et al., 2010; Lebel and Beau- 


\section{Time}

\section{Incongruent condition}

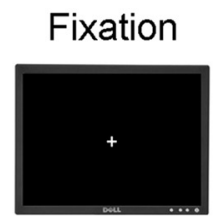

$1200-1800 \mathrm{~ms}$

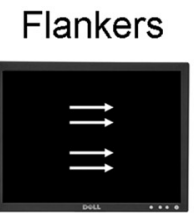

$80 \mathrm{~ms}$

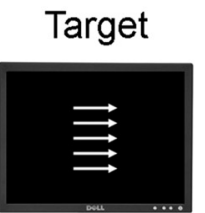

$60 \mathrm{~ms}$

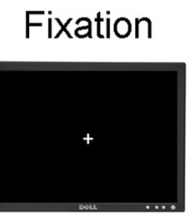

$1200-1800 \mathrm{~ms}$

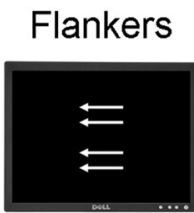

$80 \mathrm{~ms}$
Target

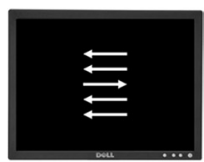

$60 \mathrm{~ms}$

Figure 1. Schematic illustration of the flanker task.

lieu, 2011). The developing brain facilitates and constrains the potential for cognitive and behavioral plasticity, but there has been virtually no examination of the neural underpinnings of performance consistency in development.

The aims of the current study were to (1) characterize developmental changes in IIV in healthy children and adolescents and (2) test the relationships between IIV and WM integrity in development. We hypothesized that IIV would decrease along with MD decrease and FA increase.

\section{Materials and Methods}

Participants. The sample was drawn from the first wave of an ongoing longitudinal project at the Center for the Study of Human Cognition, University of Oslo, called Neurocognitive Development (Østby et al., 2009; Tamnes et al., 2010a). The study was approved by the Regional Ethical Committee of South Norway. Children and adolescents were recruited though newspaper ads and local schools. Written informed consent was obtained from all participants older than 12 years of age and from a parent of participants under 18 years of age. Oral informed consent was given by participants under 12 years of age. Parents and participants aged 16-19 years were screened with separate standardized health interviews to ascertain eligibility. Participants were required to be right handed; be fluent Norwegian speakers; have normal or corrected-tonormal vision and hearing; not have a history of injury or disease known to affect CNS function, including neurological or psychiatric illness or serious head trauma; not be under psychiatric treatment; not use psychoactive drugs known to affect CNS functioning; not have had complicated or premature birth; and not have magnetic resonance imaging (MRI) contraindications. Additionally, all scans were evaluated by a neuroradiologist and required to be deemed free of significant injuries or conditions. One hundred fifteen participants satisfied these criteria. Seven participants were excluded because of incomplete or inadequate quality MRI data, and seven participants were excluded because of missing behavioral data. Nine subjects were excluded because of behavioral criteria defined in the flanker task as described below. This yielded a final sample of 92 participants (48 females) aged 8.3-19.7 years (mean, 14.3; SD, 3.4). The mean ages for females and males were 14.3 years $(\mathrm{SD}, 3.5)$ and 14.4 years $(\mathrm{SD}, 3.3)$, respectively, not significantly different. The mean IQ for the sample, as assessed by the Wechsler Abbreviated Scale of Intelligence (WASI) (Wechsler, 1999), was 108.8 (SD, 9.9; range, 91-132).

Experimental task. We administered a speeded version of the flanker task (Eriksen and Eriksen, 1974), similar to the task used by Debener et al. (2005), Westlye et al. (2009), and Fjell et al. (2011). An illustration of the task is shown in Figure 1. The stimuli were five white horizontal arrows of length approximately $1^{\circ}$, pointing either to the left or to the right, displayed in a vertical stack approximately $2.5^{\circ}$ high on a black background. Participants were to respond as accurately and quickly as possible by button presses indicating which direction the middle arrow was pointing. Each trial consisted of the following stimuli. First, a fixation cross was presented at the center of the screen for a random interval ranging between 1200 and $1800 \mathrm{~ms}$. Then, the four horizontal flanker arrows were presented for $80 \mathrm{~ms}$ above and below the screen center, before the middle target arrow was presented for $60 \mathrm{~ms}$ along with the flanker arrows. Finally, a black screen was presented for up to $1440 \mathrm{~ms}$. The flanker arrows were presented before the target to increase prepotent responding and task difficulty. At the presentation of the target, the task was to push one button if the target was pointing to the left and another button if the target was pointing to the right. Based on the mean RT for the 20 first consecutive trials, an individually adjusted RT criterion was set (mean RT $\times 1.15$ ). After every subsequent third trial with either RT exceeding this criterion or omission of response, a feedback was presented on the screen for $1 \mathrm{~s}$ instructing the participant to try to respond faster. This was implemented to increase the demand to respond quickly and thus also increase task difficulty.

There were two experimental task conditions, congruent and incongruent. In the congruent condition, the flanker arrows pointed in the same direction as the target arrow. In the incongruent condition, the flanker arrows pointed in the opposite direction of the target arrow. The probability of an incongruent trial was 50\% in a randomized fashion. The task included 416 trials with a short break halfway. Before the experimental trials began, participants completed two practice blocks of 12 trials each. In the first practice block, both the flankers and the target arrow were presented for slightly longer intervals (150 and 90 $\mathrm{ms}$, respectively), whereas in the second block, the intervals were the same as in the experimental trials. The experimental procedure was administered using E-prime software (Schneider et al., 2002), and responses were obtained on a PST Serial Response Box.

This and similar tasks have been shown to produce the well described congruency effect, with a higher RT and lower accuracy in the incongruent compared with the congruent condition (Eriksen and Eriksen, 1974). The congruency effect probably reflects response conflict induced in the incongruent condition (Botvinick et al., 2001). Responses to incongruent stimuli reflect more complex cognitive processing, including inhibitory processes, top-down controlled attention, and possibly different response strategies, than the cognitively simpler responses to the congruent stimuli. Data from the two conditions are therefore analyzed separately.

Behavioral exclusion criteria were used in the flanker task to exclude participants with suboptimal motivation or task focus. First, no participants with $<80 \%$ accuracy in the congruent trials were included. Second, participants with a nonsignificant congruency effect on RT in correct trials were excluded (independent sample $t$ tests, incongruent RT $>$ congruent RT, $p>0.05$ ). The first criterion led to the exclusion of eight participants, and one more was excluded on the basis of the second criterion. Six of the eight participants excluded because of the accuracy criterion would also have been excluded because of the RT criterion alone. In summary, nine participants were excluded on the basis of the behavioral criteria. All data reported are based on the remaining 92 participants.

RT was recorded from each of the 416 trials. For statistical analyses, we excluded the 10 trials with the fastest and the slowest RTs for each participant. This was done as it is difficult to decide whether extreme responses represent variations in cognitive processes, or whether they result from random noise caused by factors such as the participant miss- 
ing the button, having a single lapse of attention during the course of the task, etc. Excluding the extreme ends of the RT distribution is a simple way of reducing the possibility that such noise contaminates the data, without biasing the results in either direction. Furthermore, trials following an error response were excluded, because of the phenomenon of posterror slowing possibly affecting the results. For the resulting correct trials, median RT (mRT) and the SD of the RT (sdRT) were calculated and used as the main measures of interest. mRT was used instead of mean because RT generally does not follow strict normal distribution but has a thicker tail of slow compared with fast responses.

DTI acquisition. Imaging data were collected using a 12-channel head coil on a $1.5 \mathrm{~T}$ Siemens Avanto scanner (Siemens Medical Solutions). The pulse sequence used for diffusionweighted imaging was a single-shot, twicerefocused spin echo echoplanar imaging pulse sequence with 30 diffusion-sensitized gradient directions and the following parameters: repetition time/echo time, $8200 \mathrm{~ms} / 82 \mathrm{~ms}$; $b$ value, $700 \mathrm{~s} / \mathrm{mm}^{2}$; voxel size, $2.0 \times 2.0 \times 2.0 \mathrm{~mm} ; 64$ axial slices. This sequence is optimized to minimize eddy current-induced image distortions (Reese et al., 2003). The sequence was repeated in two successive runs with $10 b=0$ and 30 diffusion-weighted images collected per acquisition. The total scanning time was $11 \mathrm{~min}$, $21 \mathrm{~s}$. The two acquisitions were combined during postprocessing to increase the signal-tonoise ratio. All acquisitions were visually inspected for imaging artifacts (e.g., attributable to subject motion, susceptibility artifacts, etc.).

DTI analysis. Image analyses and tensor calculations were done using FSL (www.fmrib.ox.ac.uk/ fsl/index.html) (Smith et al., 2004; Woolrich et al., 2009). First, each DTI volume was affine registered to the T2-weighted $b=0$ volume using FLIRT (FMRIB's Linear Image Registration Tool) (Jenkinson and Smith, 2001). This corrected for motion between scans and residual eddy-current distortions present in the diffusion-weighted images. To preserve the orientation information after motion correction, we reoriented each volume's B matrix by applying the corresponding transformational matrix from the motioncorrection procedure. After removal of nonbrain tissue (Smith, 2002), leastsquare fits were performed to estimate the FA, eigenvector, and eigenvalue maps. MD was defined as the mean of all three eigenvalues $\left[\left(\lambda_{1}+\lambda_{2}\right.\right.$ $\left.\left.+\lambda_{3}\right) / 3\right]$, $\mathrm{AD}$ as the principal diffusion eigenvalue $\left(\lambda_{1}\right)$, and $\mathrm{RD}$ as the mean of the second and third eigenvalues $\left[\left(\lambda_{2}+\lambda_{3}\right) / 2\right]$.

Next, all individuals' FA volumes were skeletonized and transformed into a common space as used in Tract-Based Spatial Statistics (Smith et al., 2006, 2007). Briefly, all volumes were nonlinearly warped to the FMRIB58_FA template supplied with FSL, by use of local deformation procedures performed by FMRIB's Non-Linear Image Registration Tool (FNIRT) (www.fmrib.ox.ac.uk/fsl/fnirt/index.html), a nonlinear registration toolkit using a $b$-spline representation of the registration warp field (Rueckert et al., 1999). The common template used in the present study is a high-resolution average of 58 FA volumes from healthy male and female subjects aged $20-50$ years. All warped FA volumes were visually inspected for accuracy, which is especially pertinent when analyzing datasets with broad age ranges with relatively large interindividual variability in brain size and architecture. We have previously shown that FNIRT performed the native-to-standard warping adequately across age groups, including children and adolescents (Westlye et al., 2010). Next, a mean FA volume of all subjects was generated and thinned to create a mean FA skeleton representing the centers of all common tracts. We thresholded and binarized the mean skeleton at $\mathrm{FA}>0.20$ to reduce the likelihood of partial
Table 1. Task performance in speeded arrow flanker task

\begin{tabular}{|c|c|c|c|c|c|c|}
\hline & \multicolumn{3}{|c|}{ Congruent trials } & \multicolumn{3}{|c|}{ Incongruent trials } \\
\hline & Mean SD & Minimum & Maximum & Mean SD & Minim & Maximum \\
\hline Accuracy (\%) & 95.84 .4 & 81.3 & 100 & 74.814 .9 & 32.2 & 100 \\
\hline Induded correct trials $(n)$ & 162.821 .1 & 107 & 196 & 128.138 .5 & 35 & 200 \\
\hline RT mean correct trials (ms) & 398.481 .4 & 266.7 & 654.6 & 482.072 .3 & 359.0 & 724.3 \\
\hline RT median correct trials (ms) & 389.080 .3 & 260 & 638 & 479.966 .1 & 386 & 686 \\
\hline RT SD correct trials & 77.028 .1 & 29.5 & 151.4 & 66.829 .3 & 27.2 & 152.3 \\
\hline
\end{tabular}

Figure 2. Scatterplots of $m R T$ and $s d R T$ in congruent and incongruent trials against age. The correlation coefficients from the partial correlations, controlling for the effects of sex, are shown.

voluming in the borders between tissue classes, yielding a mask of 133,704 WM voxels. Individual FA values were warped onto this mean skeleton mask by searching perpendicular from the skeleton for maximum FA values. Using maximum FA values from the centers of the tracts further minimizes confounding effects attributable to partial voluming (Smith et al., 2006). The resulting tract invariant skeletons for each participant were fed into voxelwise permutation-based cross-subject statistics. Similar warping and analyses were used on $\mathrm{MD}, \mathrm{AD}$, and $\mathrm{RD}$ data sampled from voxels with FA $>0.20$.

Last, binary masks based on probabilistic WM atlases (Wakana et al., 2004; Mori et al., 2005; Hua et al., 2008) were created with a probability threshold of $5 \%$, chosen to accommodate interindividual variation in WM architecture. Eight major WM tracts in each hemisphere [anterior thalamic radiation (ATR), cingulum gyrus, cingulum hippocampus gyrus, corticospinal tract (CST), inferior fronto-occipital fasciculus, inferior longitudinal fasciculus, superior longitudinal fasciculus (SLF), uncinate fasciculus (UF)] and two commissural tracts (forceps minor, forceps major) were chosen as regions of interest (ROIs), in addition to three ROIs in the corpus callosum (CC; genu, body, splenium). Voxels intersecting both the FA skeleton and the ROIs were used for tract-wise analyses.

Statistical analyses. Several approaches exist for calculating intraindividual trial-to-trial variability, including intraindividual SD and intraindividual coefficient of variation (SD/M) (Lövden et al., 2007). In the present study, sdRT based on correct congruent and correct incongruent 


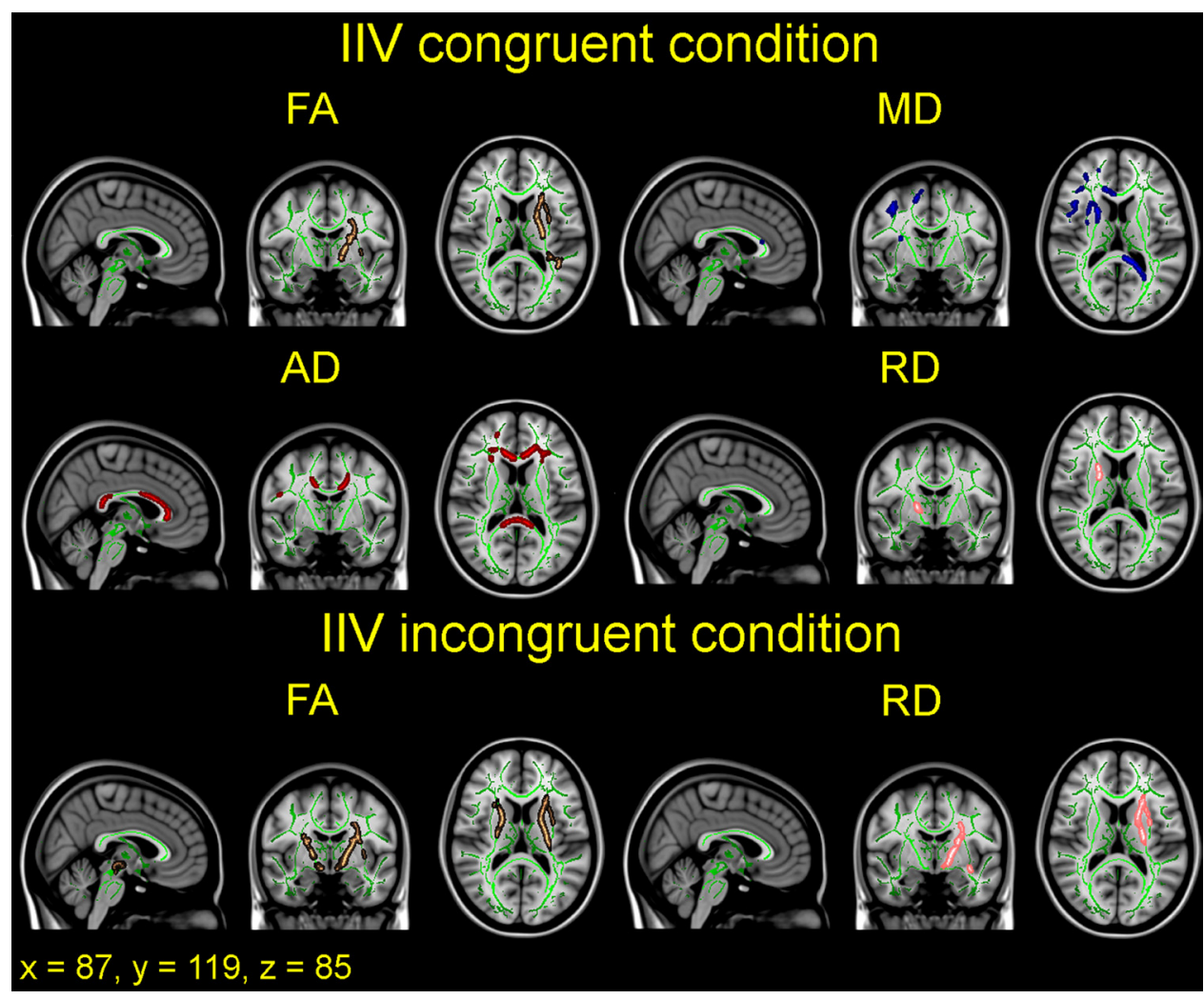

Figure 3. Overview of effects of intraindividual variability on diffusion indices. IIV was quantified as sdRT for each participant and related to diffusion anisotropy (FA) and degree of diffusion (MD, AD, RD). Sex, age, and mRT were used as covariates in the analyses. The results are corrected for multiple comparisons across space by TFCE at $p<0.05$ and are shown in selected sagittal, coronal, and horizontal views for both congruent and incongruent trials. The effects are thickened to ease visualization and are displayed on top of the green WM skeleton and the standard MNI152 T1 template. Copper, FA; blue, MD; red, AD; pink, RD. sdRT was negatively related to FA in both conditions; positively related to MD, $A D$, and RD in the congruent condition; and positively related to RD in the incongruent condition. All images are shown in radiological convention.

trials was used, as this allowed us to test the effects of IIV both with and without covarying for mRT. Initial analyses of the behavioral data were performed with descriptive statistics separately for the congruent and the incongruent trials, and independent sample $t$ tests were used to test for possible sex differences in task performance. Next, analyses on the relationships between accuracy, mRT, sdRT, and intellectual ability scores were done using partial correlations, controlling for the effects of sex and age. Third, to test for relationships between task performance and age, we performed partial correlations, controlling for the effects of sex. The analyses on the relationships between sdRT and intellectual abilities and age, respectively, were repeated while additionally controlling for the effects of mRT.

Voxelwise DTI analyses were performed using nonparametric permutation-based testing (Nichols and Holmes, 2002) as implemented in Randomise in FSL. First, we tested for linear effects of mRT on FA, $\mathrm{MD}, \mathrm{AD}$, and $\mathrm{RD}$ across the WM skeleton with general linear models (GLM) while using sex and age as covariates. Second, we tested for effects of sdRT on the four diffusion parameters while including sex, age, and $\mathrm{mRT}$ as covariates. mRT was used as a covariate in the analyses of the effects of sdRT because a correlation between $\mathrm{mRT}$ and sdRT is expected. Threshold-free cluster enhancement (TFCE) (Smith and Nichols, 2009) was used to avoid defining arbitrary cluster-forming thresholds and smoothing levels. The data were tested against an empirical null distribution generated by 10,000 permutations for each contrast, thus providing statistical maps fully corrected for multiple comparisons across space. Corrected $p<0.05$ was considered significant. To illustrate the strength of the relationships between $\mathrm{mRT}$ and sdRT, respectively, and the diffusion parameters, we performed partial correlations with averaged DTI indices in ROIs described above. In the partial correlations with mRT, we partialed out sex and age, whereas in the correlations with sdRT, sex, age, and $\mathrm{mRT}$ were controlled for.

\section{Results}

Task performance

Descriptive statistics on task performance in the congruent and incongruent conditions are shown in Table 1. For the RT measures, only correct responses were included. As expected, accuracy on incongruent trials was lower than on congruent trials, and mean and median RT were longer (Table 1). This indicates that the experimental paradigm yielded the expected effects for the included participants. There was also a difference in sdRT in the two conditions, with lower sdRT in incongruent trials. There were no significant sex differences for any of the task performance measures.

For the congruent trials, partial correlations with accuracy, controlling for sex and age, showed a negative association for $\operatorname{sdRT}\left(r=-0.40 ; p<10^{-5}\right)$ but no significant association for $\operatorname{mRT}(r=0.16 ; p=0.135)$. For the incongruent trials, the reverse was found, with no significant association for sdRT $(r=-0.11$; $p=0.320)$ but a significant positive association for mRT $(r=$ $\left.0.58 ; p<10^{-9}\right)$. Partial correlations between $\mathrm{mRT}$ and sdRT, controlling for the effects of sex and age, showed a strong positive relationship for congruent trials $\left(r=0.63 ; p<10^{-11}\right)$ and a moderate association for incongruent trials $\left(r=0.35 ; p<10^{-4}\right)$.

\section{Relationships with intellectual abilities}

To test for relationships between mRT and sdRT and general cognitive function, we performed partial correlations with intellectual abilities as indexed by the average of $z$-transformed raw performance scores on the four WASI tests (vocabulary, similar- 


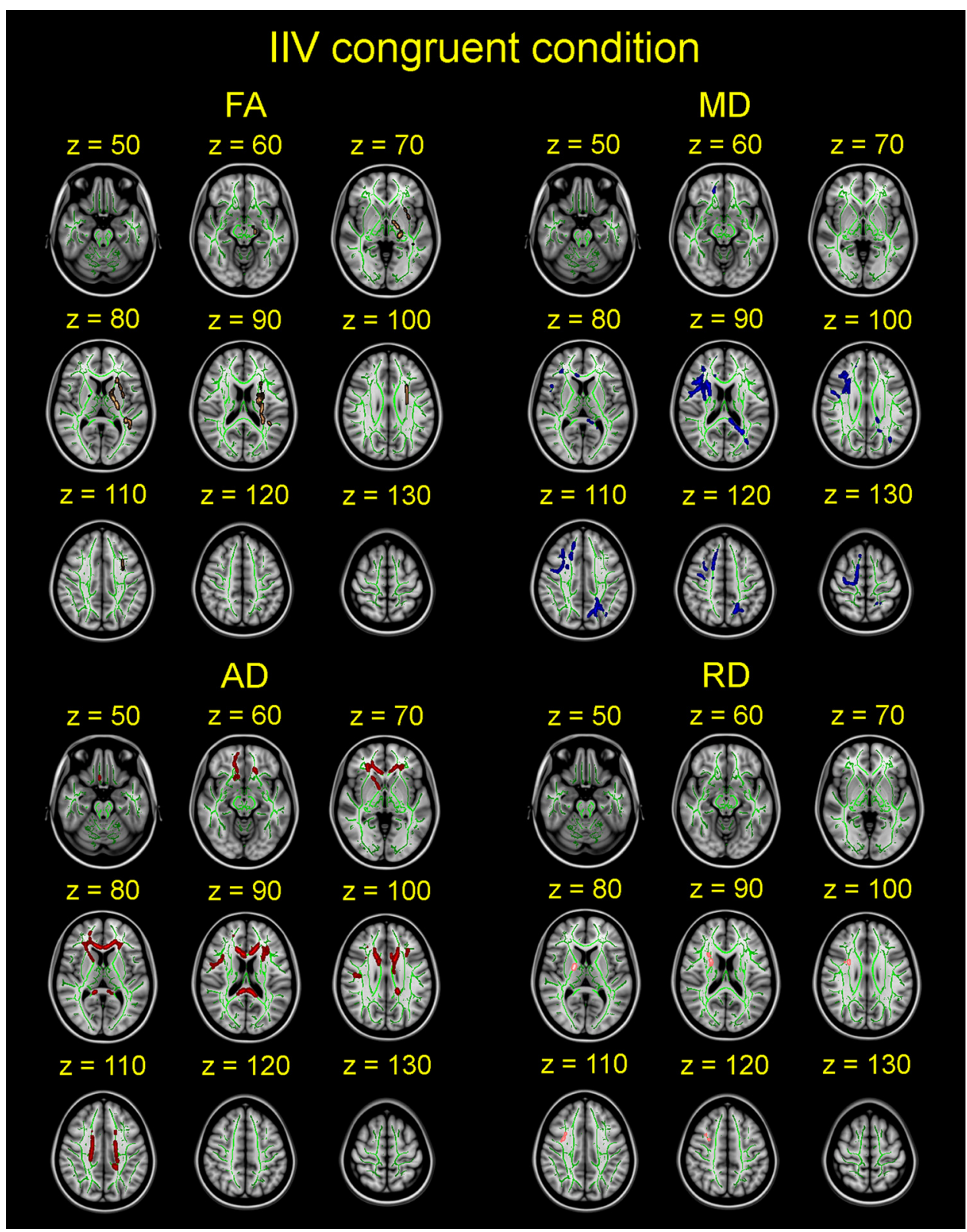

Figure 4. Effects of intraindividual variability on diffusion parameters in the congruent condition. Effects of sdRT in congruent trials on diffusion anisotropy (FA) and degree of diffusion (MD, AD, $\mathrm{RD}$ ) are shown slice by slice in the horizontal plane. Sex, age, and mRT were used as covariates, and the results are corrected for multiple comparisons across space. sdRT was negatively related to FA and positively related to $M D, A D$, and $R D$. All images are shown in radiological convention.

ities, matrix reasoning, block design), controlling for the effects of sex and age. mRT showed no relationship with intellectual abilities in the congruent $(r=-0.04 ; p=0.692)$ or the incongruent $(r=-0.02 ; p=0.831)$ condition, whereas sdRT showed a small nonsignificant negative association in both the congruent $(r=-0.14 ; p=0.185)$ and the incongruent $(r=-0.15 ; p=$ $0.158)$ conditions. Similar associations between sdRT and intellectual abilities were observed when additionally controlling for the effects of mRT (congruent trials: $r=-0.15, p=0.168$; incongruent trials: $r=-0.15, p=0.156$ ).

Relationships with age

The relationships between task performance and age were tested by partial correlations, controlling for the effects of sex. The percentage of correct congruent trials was positively related to age $\left(r=0.53 ; p<10^{-8}\right)$, whereas correct incongruent trials was not $(r=0.06 ; p=0.577)$. mRT showed strong negative relationships with age in both the congruent $\left(r=-0.63 ; p<10^{-11}\right)$ and the incongruent $\left(r=-0.66 ; p<10^{-12}\right)$ conditions. For sdRT, we found even stronger negative associations with age in both the congruent $\left(r=-0.69 ; p<10^{-14}\right)$ and the incongruent $(r=$ $\left.-0.73 ; p<10^{-16}\right)$ conditions. Scatterplots of mRT and sdRT in the two conditions by age are shown in Figure 2. When controlling for mRT in addition to sex, sdRT was still negatively correlated with age in both the congruent $\left(r=-0.41 ; p<10^{-5}\right)$ and the incongruent $\left(r=-0.52 ; p<10^{-7}\right)$ condition.

Relationships with DTI parameters

We tested for linear effects of mRT and sdRT, respectively, on DTI indices on a voxel-by-voxel basis with GLMs. Sex and age were included as covariates in all analyses, and mRT was included as an additional covariate in all analyses involving sdRT. There 


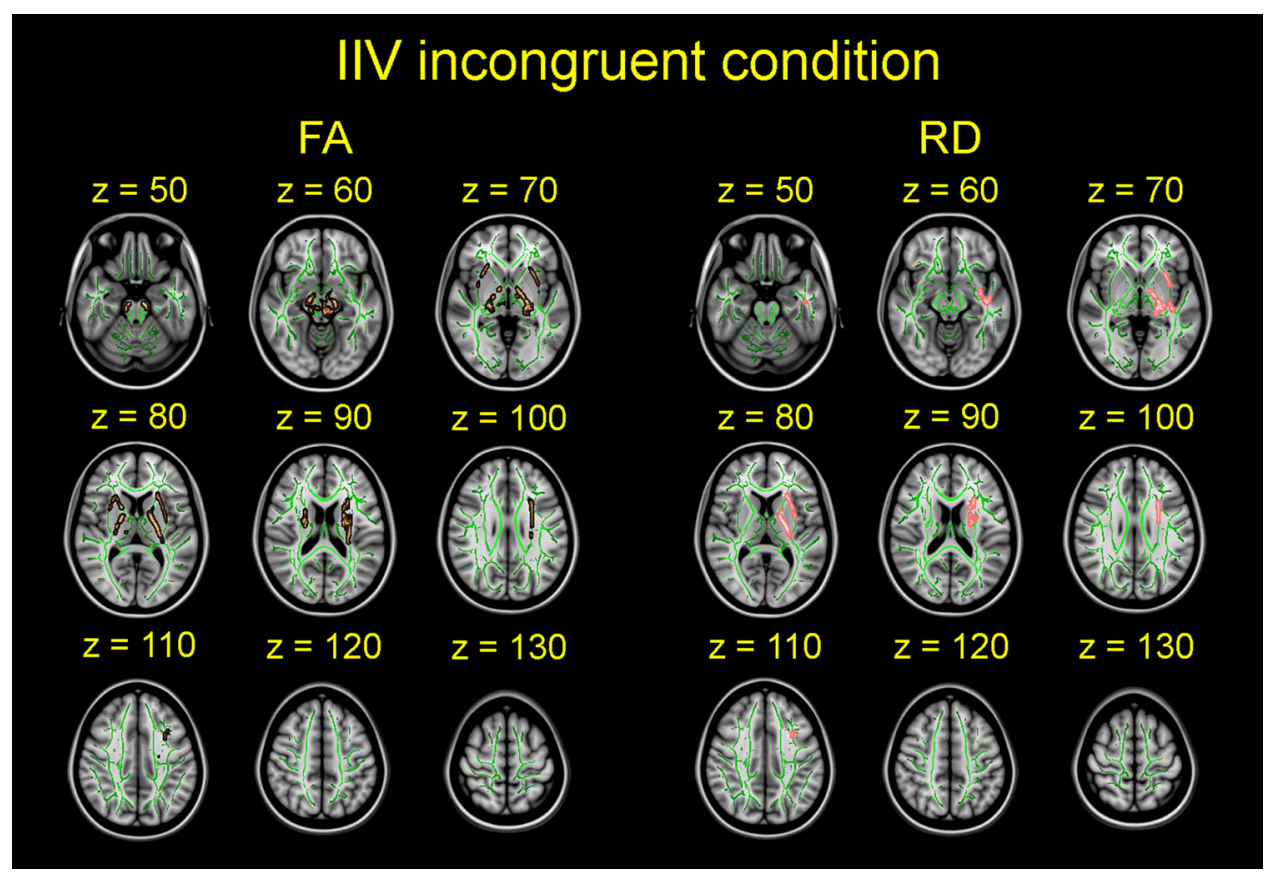

Figure 5. Effects of intraindividual variability on diffusion parameters in the incongruent condition. Effects of sdRT in incongruent trials on diffusion anisotropy (FA) and RD are shown slice by slice in the horizontal plane. Sex, age, and mRT were used as covariates, and the effects are corrected for multiple comparisons across space. sdRT was negatively related to FA and positively related to RD. All images are shown in radiological convention.

were no significant associations between $\mathrm{mRT}$ and $\mathrm{FA}, \mathrm{MD}, \mathrm{AD}$, or RD in either the congruent or the incongruent condition. In contrast, lower sdRT was significantly associated with higher FA in both conditions; lower $\mathrm{MD}, \mathrm{AD}$, and $\mathrm{RD}$ in the congruent condition; and lower RD in the incongruent condition. An overview of the significant relationships between sdRT in both conditions and the DTI parameters is shown in three views in Figure 3. The same results, slice by slice, in the horizontal plane for nine slices for each DTI parameter are shown in Figures 4 and 5 for the congruent and the incongruent conditions, respectively. The results are described in more detail below.

For the congruent trials, lower sdRT was associated with higher FA and lower MD, AD, and RD. No significant effects were observed in the opposite directions. For FA, significant associations were found in $1.98 \%$ ( 2649 of the total 133,704 ) of the WM skeleton voxels. The areas of effects included central sections in the left hemisphere around the CST and the SLF and a small region around the right CST. Note, however, that the uncorrected results did not show evidence of strong asymmetry (see also ROI analyses below). For MD, associations were found in $4.27 \%$ ( 5712 voxels) of the skeleton voxels, and the areas of effects were more widespread, including mainly posterior sections in the left hemisphere and anterior sections in the right hemisphere. For $\mathrm{AD}, 5.18 \%$ (6922 voxels) of the skeleton voxels showed significant associations with sdRT. The effects were located in frontal sections in both hemispheres and in both frontal and posterior sections in and extending from the CC. RD was associated with sdRT in $0.37 \%$ (497 voxels) of the skeleton voxels in a central section in the right hemisphere, mainly around the CST.

For the incongruent trials, lower sdRT was associated with higher FA and lower RD. No significant effects were observed on $\mathrm{MD}$ or $\mathrm{AD}$ or in the opposite directions on FA or RD. For FA, associations were found in $4.38 \%$ ( 5854 voxels) of the WM skeleton voxels. As for the congruent trials, the areas of effects encompassed central sections around the CST and the SLF; however, the significant effects in the incongruent trials were bilateral. For RD, 1.75\% (2341 voxels) of the skeleton voxels showed significant associations with sdRT. The areas of effects were thus more extensive than on $\mathrm{RD}$ for the congruent trials and were mainly in central areas in the left hemisphere.

To illustrate the strength of the relationships between IIV and WM integrity, we performed partial correlations between sdRT and the mean DTI values in the ROIs, controlling for the effects of sex, age, and mRT. The results are presented in Table 2. Briefly, the strongest associations between sdRT and the tract-wise DTI indices were seen for selected diffusion parameters in one or both conditions bilaterally in the CST and the UF, in the left SLF, in forceps minor, and in the genu and splenium of the CC. The effect sizes of these associations were of small to moderate magnitude. Scatterplots illustrating the raw data for selected areas are presented in Figure 6 for the congruent trials and in Figure 7 for the incongruent trials. For comparison purposes, we also performed partial correlations between mRT and the mean DTI values in the ROIs, controlling for the effects of sex and age. The only uncorrected significant association was found in the genu of the CC, where $\mathrm{mRT}$ unexpectedly was positively related to FA in both the congruent $(r=0.30 ; p=0.004)$ and the incongruent $(r=$ $0.23 ; p=0.030)$ conditions and negatively related to $\mathrm{RD}$ in the congruent $(r=-0.26 ; p=0.013)$ condition. Note, however, that there were no significant effects of $\mathrm{mRT}$ on $\mathrm{FA}, \mathrm{MD}, \mathrm{AD}$, or $\mathrm{RD}$ in either condition in the voxelwise corrected analyses above.

\section{Discussion}

In the present study, we have characterized age-related reductions in within-person variability of response speed in children and adolescents and have demonstrated significant relationships between performance consistency and DTI indices of WM integrity in development for the first time. The behavioral changes and the relationships with WM integrity are discussed in more detail below. 
Table 2. Partial correlations between sdRT and DTI parameters

\begin{tabular}{|c|c|c|c|c|c|c|c|c|}
\hline & \multicolumn{4}{|c|}{ Congruent trials } & \multicolumn{4}{|c|}{ Incongruent trials } \\
\hline & $\mathrm{FA}$ & MD & $A D$ & $\mathrm{RD}$ & $\mathrm{FA}$ & MD & $A D$ & $\mathrm{RD}$ \\
\hline Anterior thalamic radiation $\mathrm{L}$ & -0.09 & 0.03 & 0.03 & 0.02 & -0.18 & 0.10 & 0.04 & 0.12 \\
\hline Anterior thalamic radiation $\mathrm{R}$ & 0.02 & 0.02 & 0.11 & -0.03 & -0.07 & 0.05 & 0.06 & 0.04 \\
\hline Cingulum gyrus $\mathrm{R}$ & -0.03 & 0.09 & 0.07 & 0.08 & -0.03 & 0.02 & -0.01 & 0.03 \\
\hline Cingulum hippocampus gyrus L & -0.04 & 0.09 & 0.07 & 0.09 & 0.04 & 0.04 & 0.07 & 0.01 \\
\hline Cingulum hippocampus gyrus $\mathrm{R}$ & -0.00 & -0.01 & -0.01 & -0.02 & 0.11 & -0.11 & -0.06 & -0.13 \\
\hline Inferior fronto-occipital fasciculus $\mathrm{L}$ & -0.12 & 0.17 & 0.13 & 0.16 & -0.17 & 0.16 & 0.08 & 0.18 \\
\hline Inferior fronto-occipital fasciculus $R$ & -0.00 & 0.13 & 0.17 & 0.08 & -0.04 & 0.09 & 0.09 & 0.08 \\
\hline Inferior longitudinal fasciculus L & -0.19 & 0.15 & 0.07 & 0.18 & -0.15 & 0.14 & 0.08 & 0.16 \\
\hline Inferior longitudinal fasciculus R & -0.04 & 0.10 & 0.10 & 0.02 & -0.01 & 0.04 & 0.04 & 0.03 \\
\hline Superior longitudinal fasciculus $\mathrm{L}$ & -0.24 & 0.19 & 0.11 & 0.22 & -0.16 & 0.16 & 0.11 & 0.17 \\
\hline Superior longitudinal fasciculus $R$ & -0.19 & 0.18 & 0.15 & 0.18 & -0.15 & 0.14 & 0.10 & 0.16 \\
\hline Corpus callosum body & -0.01 & 0.12 & 0.16 & 0.06 & -0.08 & 0.11 & 0.07 & 0.10 \\
\hline Corpus callosum splenium & -0.15 & 0.21 & 0.16 & 0.18 & -0.10 & 0.10 & 0.07 & 0.10 \\
\hline
\end{tabular}

Tracts and regions were defined by probabilistic atlases in FSL. The effects of sex, age, and mRT were controlled for. Uncorrected significant $(p<0.05)$ correlations are in bold. L, Left; R, right.
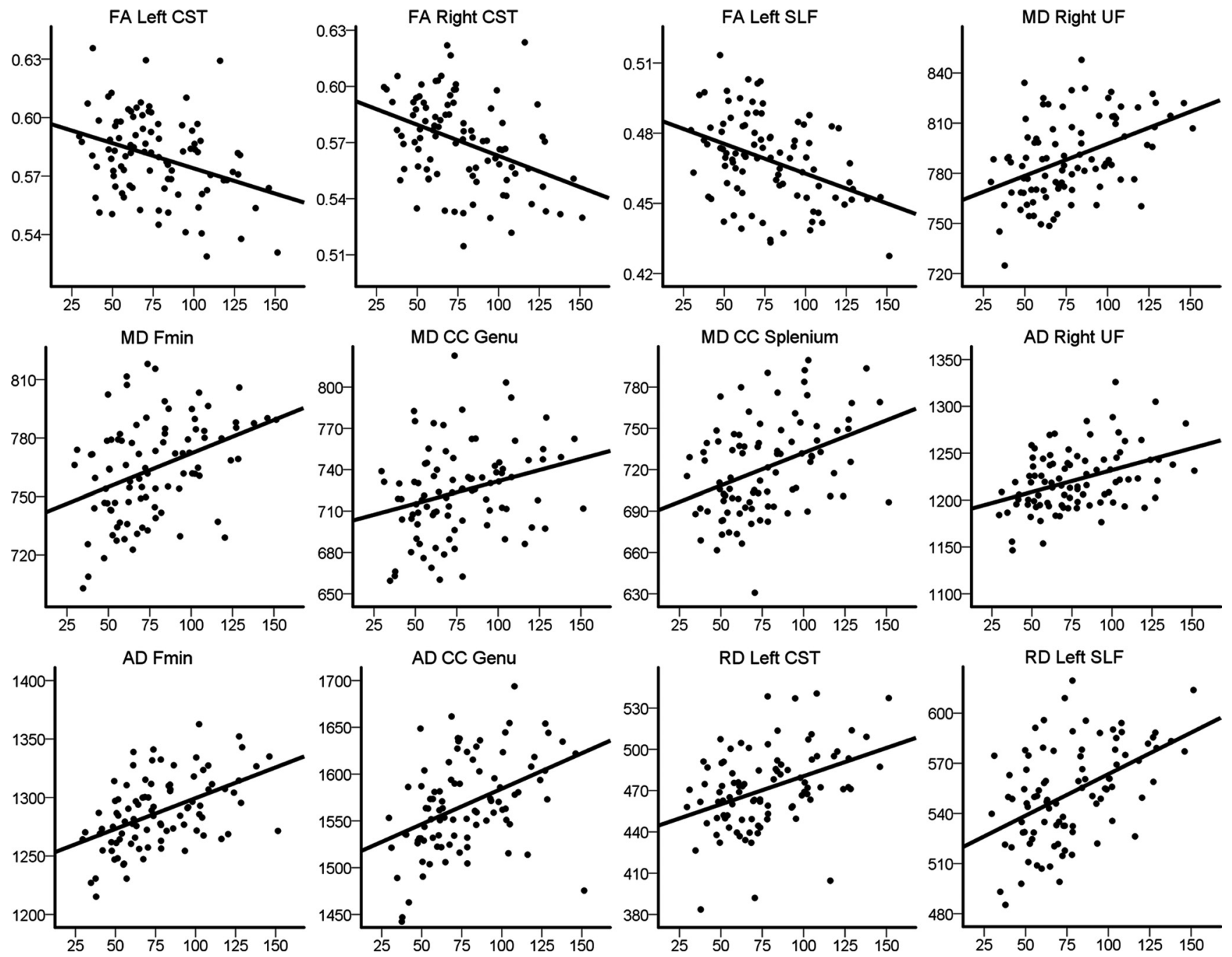

Figure 6. Scatterplots of intraindividual variability in congruent trials and diffusion indices in selected regions. The scatterplots show sdRT in congruent trials against FA, MD, AD, and RD in selected areas. The plots are used to illustrate the raw data, and thus sex, age, and mRT are not used as covariates, in contrast to the statistical analyses presented in Table 2. Fmin, Forceps minor. 


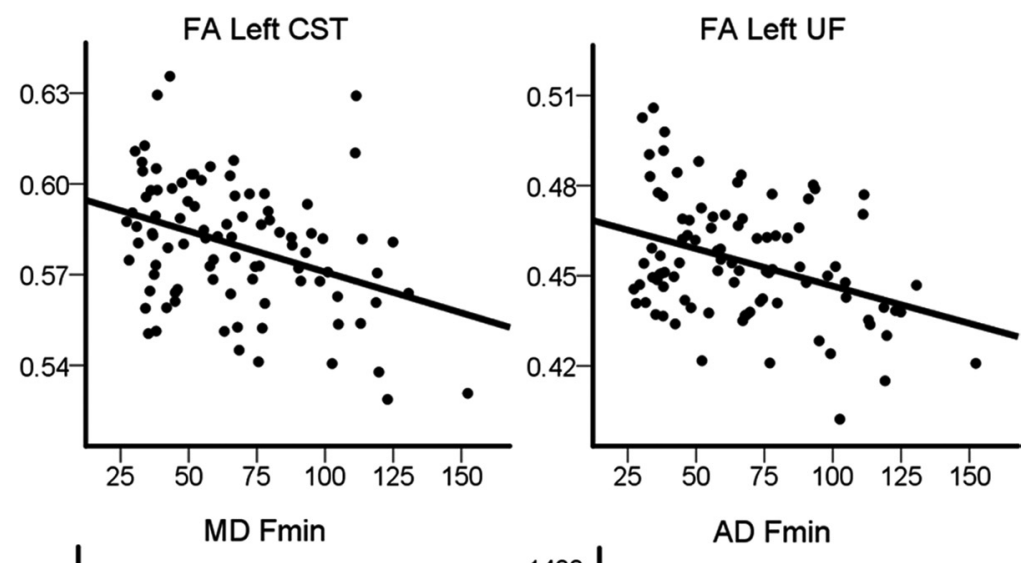

Research on the cognitive origins of IIV remains sparse, although several candidate mechanisms have been postulated to account for increased IIV in aging, including momentary lapses of attention and failures to maintain executive control (Bunce et al., 1993; MacDonald et al., 2009; West et al., 2002). It is, however, likely that the large IIV in children and older adults reflects at least partly different mechanisms (Fjell et al., 2007). Brewer and Smith (1989) postulated that increased ability to monitor and regulate speed-accuracy tradeoffs in RT tasks causes the reductions in $\mathrm{RT}$ seen during development and that the poorer regulation in children causes larger IIV. Interestingly, in a recent study of 7 and 9 year olds, less variable error RTs was related to stronger amplitude of the errorrelated negativity, an electrophysiological marker of performance monitoring (Richardson et al., 2011). A number of cognitive processes could, however, underlie the developmental changes in IIV, and it is less likely that IIV reflects one unitary cognitive mechanism. Rather, cognitive variability may reflect different antecedents depending on task, practice (Allaire and Marsiske, 2005), and time scale (Li et al., 2004a; Schmiedek et al., 2009). Additional studies with more complex designs, and including other behavioral measures, are required to separate different possible cognitive mechanisms underlying IIV and to directly test the relationships between developmental reductions in IIV and improvements in specific cognitive functions.

Despite its theoretical significance,

Figure 7. Scatterplots of intraindividual variability in incongruent trials and diffusion indices in selected regions. The scatterplots show sdRT in incongruent trials against $F A, M D, A D$, and RD in selected areas. The plots are used to illustrate the raw data, and thus sex, age, and mRT are not used as covariates (see Table 2 for partial correlation coefficients). Fmin, Forceps minor.

there have, to date, been a limited number of studies relating within-person variability to brain characteristics and no such

Large developmental reductions in IIV of correct RTs were found, in agreement with previous life-span studies (Li et al., 2004b; Williams et al., 2005). The developmental reduction of IIV was established independently of age-related differences in response speed and for both simple congruent trials and more complex incongruent trials. Increased IIV has been shown to characterize performance of older adults and populations with various conditions, including traumatic brain injury, dementia, and schizophrenia (MacDonald et al., 2006). Furthermore, increased IIV may reflect core neuronal deficiencies in children with attention-deficit hyperactivity disorder (Castellanos and Tannock, 2002; Bellgrove et al., 2005; Aase et al., 2006), but few studies have focused on developmental differences per se. Notably, we found larger age-related reductions for response speed variability than for average response speed, indicating that IIV could be a sensitive behavioral marker of cognitive development. However, sdRT showed small nonsignificant associations with general intellectual abilities, whereas no associations were found between $\mathrm{mRT}$ and intellectual abilities. studies on children or adolescents. Independently of age and average speed of responding, we found that lower IIV was associated with higher FA in both task conditions; lower MD, $\mathrm{AD}$, and $\mathrm{RD}$ in the congruent trials; and lower RD in the incongruent trials. It is reasonable to hypothesize that such age-independent associations are at least partly mediated by developmental variability, i.e., variability among children of similar age in the phase of WM maturation (Jernigan et al., 2011). Thus, children with more mature WM will, in general, exhibit lower degrees of performance variability. From childhood to adult age, the brain undergoes dynamic structural changes (Giedd and Rapoport, 2010). FA increases and overall diffusivity decreases indicate maturation of WM integrity (Bava et al., 2010; Giorgio et al., 2010; Lebel and Beaulieu, 2011) and are believed to be related to developmental changes in myelin content, axon caliber and/or neurite density, and changes in gross fiber organization (Paus, 2010). There is evidence that DTI provides promising WM microstructural phenotypes related to cognitive development (Olson et al., 2009; Tsang et al., 2009; Johansen-Berg, 2010; Madsen et al., 2010; 
Tamnes et al., 2010b). The present results provide novel evidence that DTI indices are related to performance consistency in development and support the proposition that developmental reductions in within-person performance variability reflect maturation of WM connectivity.

Neuropsychological studies on patients with focal lesions (Stuss et al., 2003) and dementia (Murtha et al., 2002) have suggested an association between within-person variability and frontal brain structures. The frontal lobe hypothesis has also been partly supported by a functional MRI study that found an association between IIV and task-related brain activation within a network consisting of middle frontal, inferior parietal, and thalamic regions (Bellgrove et al., 2004). An association between IIV and frontal WM integrity could be expected in adolescence, as regional differences observed in developmental curves indicate a pattern of maturation in which fronto-temporal networks show a relatively protracted development (Tamnes et al., 2010a; Colby et al., 2011; Lebel and Beaulieu, 2011). However, as discussed below, the majority of effects found in the current study were in central brain regions.

To our knowledge, six structural imaging studies have investigated the neuroanatomical correlates of IIV in adults. Anstey et al. (2007) found a negative association between CC area and IIV in individuals with mild cognitive disorders but minimal associations in healthy aging individuals. However, Bunce et al. (2007) found that IIV was associated with frontal lobe WM hyperintensities in healthy older adults. Furthermore, negative relationships between IIV and total WM volume (Walhovd and Fjell, 2007) and WM volume in frontal, parietal, and temporal regions (Ullen et al., 2008) have been reported. Recently, Moy et al. (2011) tested for associations with both gray matter density and WM integrity in 61 adults aged 22-88 years. Only FA showed an age-independent relationship with IIV, with negative relationships in the splenium of the CC and in the left inferior fronto-occipital fasciculus. Fjell et al. (2011) reported robust age-independent relationships between IIV and all tested DTI indices in 270 subjects aged $20-83$ years. Importantly, the two latter studies indicate that the associations between IIV and WM characteristics in adults are not restricted to the frontal lobes.

In the current study, the spatial distribution of effects was substantially smaller than in the study by Fjell et al. (2011), possibly because of lower statistical power and more variance explained by age. Nonetheless, the associations between IIV and diffusion parameters were not predominantly in frontal regions but in central sections, including the CST and both the genu and splenium of the CC. The CST is a descending pathway mainly from the precentral motor cortex into the brain stem, whereas CC is the main interhemispheric pathway. However, effects were also seen in forceps minor, bilaterally in the UF and in the left SLF, all pathways with frontal connections.

We found no significant age-independent relationships between response speed $(\mathrm{mRT})$ and DTI indices. In a recent study including children aged 7-13, faster choice RT was associated with lower MD within the CST and neostriatum (Madsen et al., 2011). One reason for this discrepancy is likely that we, in the present study, included age as a covariate and that mRT and age were highly correlated, suggesting a common effect of age. In addition, the results may be affected by a number of factors, including different tasks and that the current study covered a wider age range and performed voxelwise analyses using a more conservative statistical threshold. Fjell et al. (2011) reported DTI relationships with response speed in congruent trials only with
$\mathrm{MD}$ and $\mathrm{AD}$ in the genu and body of the $\mathrm{CC}$ and with $\mathrm{AD}$ in the left ATR and forceps minor. In agreement with Fjell et al. (2011), the present results indicate that WM integrity is related stronger to response speed variability than to average response speed.

The neural mechanisms of IIV reductions in development are likely complex and multifaceted. Brain function variability, indexed by single-trial electroencephalographic signal variability, has been found to increase with maturation and to be negatively correlated with behavioral variability (McIntosh et al., 2008). A negative relationship between behavioral variability and blood oxygen level-dependent signal variability was also recently reported (Garrett et al., 2011). It can be speculated that the emergence and shaping of structural brain connections during development allows for more integrated, distributed, and thus more variable brain responses, which at the behavioral level may manifest as increased RT consistency. A number of factors influence DTI indices (Beaulieu, 2009), and the relative roles of these factors are possibly age dependent. Importantly, DTI parameters are sensitive to general diffusion patterns and are not selective markers of specific neurobiological properties. Biological interpretations of DTI associations are thus challenging and should be done with caution.

In conclusion, we found large developmental reductions in trial-to-trial variability of RT in children and adolescents, and that level of performance consistency is associated with DTI indices of WM integrity in development. The results indicate that reduced performance variability partly reflects maturation of WM connectivity. The findings highlight the importance of considering within-person variability in theories of cognitive development and its neurobiological basis.

\section{References}

Aase H, Meyer A, Sagvolden T (2006) Moment-to-moment dynamics of ADHD behaviour in South African children. Behav Brain Funct 2:11.

Allaire JC, Marsiske M (2005) Intraindividual variability may not always indicate vulnerability in elders' cognitive performance. Psychol Aging 20:390-401.

Anstey KJ, Mack HA, Christensen H, Li SC, Reglade-Meslin C, Maller J, Kumar R, Dear K, Easteal S, Sachdev P (2007) Corpus callosum size, reaction time speed and variability in mild cognitive disorders and in a normative sample. Neuropsychologia 45:1911-1920.

Basser PJ, Jones DK (2002) Diffusion-tensor MRI: theory, experimental design and data analysis-a technical review. NMR Biomed 15:456-467.

Bava S, Thayer R, Jacobus J, Ward M, Jernigan TL, Tapert SF (2010) Longitudinal characterization of white matter maturation during adolescence. Brain Res 1327:38-46.

Beaulieu C (2002) The basis of anisotropic water diffusion in the nervous system-a technical review. NMR Biomed 15:435-455.

Beaulieu C (2009) The biological basis of diffusion anisotropy. In: Diffusion MRI (Johansen-Berg H, Behrens TE, eds), pp 105-126. Amsterdam: Elsevier.

Bellgrove MA, Hester R, Garavan H (2004) The functional neuroanatomical correlates of response variability: evidence from a response inhibition task. Neuropsychologia 42:1910-1916.

Bellgrove MA, Hawi Z, Kirley A, Gill M, Robertson IH (2005) Dissecting the attention deficit hyperactivity disorder (ADHD) phenotype: sustained attention, response variability and spatial attentional asymmetries in relation to dopamine transporter (DAT1) genotype. Neuropsychologia 43:1847-1857.

Botvinick MM, Braver TS, Barch DM, Carter CS, Cohen JD (2001) Conflict monitoring and cognitive control. Psychol Rev 108:624-652.

Brewer N, Smith GA (1989) Developmental changes in processing speed: influences of speed-accuracy regulation. J Exp Psychol Gen 118:298-310.

Bunce DJ, Warr PB, Cochrane T (1993) Blocks in choice responding as a function of age and physical fitness. Psychol Aging 8:26-33.

Bunce D, Anstey KJ, Christensen H, Dear K, Wen W, Sachdev P (2007) White matter hyperintensities and within-person variability in communitydwelling adults aged 60-64 years. Neuropsychologia 45:2009-2015. 
Castellanos FX, Tannock R (2002) Neuroscience of attention-deficit/hyperactivity disorder: the search for endophenotypes. Nat Rev Neurosci 3:617-628.

Colby JB, Van Horn JD, Sowell ER (2011) Quantitative in vivo evidence for broad regional gradients in the timing of white matter maturation during adolescence. Neuroimage 54:25-31.

Debener S, Ullsperger M, Siegel M, Fiehler K, von Cramon DY, Engel AK (2005) Trial-by-trial coupling of concurrent electroencephalogram and functional magnetic resonance imaging identifies the dynamics of performance monitoring. J Neurosci 25:11730-11737.

Eriksen BA, Eriksen CW (1974) Effects of noise letters upon the identification of a target letter in a nonsearch task. Percept Psychophys 16:143-149.

Fjell AM, Østby Y, Walhovd KB (2007) Intraindividual variability in cognitive performance and its neural foundation-an important approach in studying the developing and aging mind. In: Leading-edge psychological tests and testing research (Lange MA, ed), pp 193-200. Hauppauge, NY: Nova Science Publishers.

Fjell AM, Westlye LT, Amlien IK, Walhovd KB (2011) Reduced white matter integrity is related to cognitive instability. J Neurosci 31:18060-18072.

Fry AF, Hale S (1996) Processing speed, working memory, and fluid intelligence: evidence for a developmental cascade. Psychol Sci 7:237-241.

Garrett DD, Kovacevic N, McIntosh AR, Grady CL (2011) The importance of being variable. J Neurosci 31:4496-4503.

Giedd JN, Rapoport JL (2010) Structural MRI of pediatric brain development: what have we learned and where are we going? Neuron 67:728 -734.

Giorgio A, Watkins KE, Chadwick M, James S, Winmill L, Douaud G, De Stefano N, Matthews PM, Smith SM, Johansen-Berg H, James AC (2010) Longitudinal changes in grey and white matter during adolescence. Neuroimage 49:94-103.

Hua K, Zhang J, Wakana S, Jiang H, Li X, Reich DS, Calabresi PA, Pekar JJ, van Zijl PC, Mori S (2008) Tract probability maps in stereotaxic spaces: analyses of white matter anatomy and tract-specific quantification. Neuroimage 39:336-347.

Hultsch DF, MacDonald SW, Dixon RA (2002) Variability in reaction time performance of younger and older adults. J Gerontol Psychol Sci 57B:101-115.

Jenkinson M, Smith S (2001) A global optimisation method for robust affine registration of brain images. Med Image Anal 5:143-156.

Jernigan TL, Baaré WF, Stiles J, Madsen KS (2011) Postnatal brain development: structural imaging of dynamic neurodevelopmental processes. Prog Brain Res 189:77-92.

Johansen-Berg H (2010) Behavioural relevance of variation in white matter microstructure. Curr Opin Neurol 23:351-358.

Lebel C, Beaulieu C (2011) Longitudinal development of human brain wiring continues from childhood into adulthood. J Neurosci 31:10937-10947.

Le Bihan D (2003) Looking into the functional architecture of the brain with diffusion MRI. Nat Rev Neurosci 4:469-480.

Li SC, Huxhold O, Schmiedek F (2004a) Aging and attenuated processing robustness. Evidence from cognitive and sensorimotor functioning. Gerontology 50:28-34.

Li SC, Lindenberger U, Hommel B, Aschersleben G, Prinz W, Baltes PB (2004b) Transformations in the couplings among intellectual abilities and constituent cognitive processes across the life span. Psychol Sci $15: 155-163$

Li SC, Hämmerer D, Müller V, Hommel B, Lindenberger U (2009) Lifespan development of stimulus-response conflict cost: similarities and differences between maturation and senescence. Psychol Res 73:777-785.

Lindenberger U, von Oertzen T (2006) Variability in cognitive aging: from taxonomy to theory. In: Lifespan cognition: mechanisms of change (Bialystok E, Craik FIM, eds), pp 297-314. Oxford: Oxford UP.

Lövden M, Li SC, Shing YL, Lindenberger U (2007) Within-person trial-totrial variability precedes and predicts cognitive decline in old and very old age: longitudinal data from the Berlin Aging Study. Neuropsychologia 45:2827-2838.

MacDonald SW, Nyberg L, Bäckman L (2006) Intra-individual variability in behavior: links to brain structure, neurotransmission and neuronal activity. Trends Neurosci 29:474-480.

MacDonald SW, Li SC, Bäckman L (2009) Neural underpinnings of withinperson variability in cognitive functioning. Psychol Aging 24:792-808.

Madsen KS, Baaré WF, Vestergaard M, Skimminge A, Ejersbo LR, Ramsøy TZ, Gerlach C, Akeson P, Paulson OB, Jernigan TL (2010) Response inhibition is associated with white matter microstructure in children. Neuropsychologia 48:854-862.

Madsen KS, Baaré WF, Skimminge A, Vestergaard M, Siebner HR, Jernigan TL (2011) Brain microstructural correlates of visuospatial choice reaction time in children. Neuroimage 58:1090-1100.

McIntosh AR, Kovacevic N, Itier RJ (2008) Increased brain signal variability accompanies lower behavioral variability in development. PLoS Comput Biol 4:e1000106.

Mori S, Wakana S, Nagae-Poetscher LM, van Zijl PCM (2005) MRI atlas of human white matter. Amsterdam: Elsevier.

Moy G, Millet P, Haller S, Baudois S, de Bilbao F, Weber K, Lövblad K, Lazeyras F, Giannakopoulos P, Delaloye C (2011) Magnetic resonance imaging determinants of intraindividual variability in the elderly: combined analysis of grey and white matter. Neuroscience 186:88-93.

Murtha S, Cismaru R, Waechter R, Chertkow H (2002) Increased variability accompanies frontal lobe damage in dementia. J Int Neuropsychol Soc 8:360-372.

Nichols TE, Holmes AP (2002) Nonparametric permutation tests for functional neuroimaging: a primer with examples. Hum Brain Mapp 15:1-25.

Olson EA, Collins PF, Hooper CJ, Muetzel R, Lim KO, Luciana M (2009) White matter integrity predicts delay discounting behavior in 9- to $23-$ year-olds: a diffusion tensor imaging study. J Cogn Neurosci 21:1406-1421.

Østby Y, Tamnes CK, Fjell AM, Westlye LT, Due-Tønnessen P, Walhovd KB (2009) Heterogeneity in subcortical brain development: a structural magnetic resonance imaging study of brain maturation from 8 to 30 years. J Neurosci 29:11772-11782.

Paus T (2010) Growth of white matter in the adolescent brain: myelin or axon? Brain Cogn 72:26-35.

Reese TG, Heid O, Weisskoff RM, Wedeen VJ (2003) Reduction of eddycurrent-induced distortion in diffusion MRI using a twice-refocused spin echo. Magn Reson Med 49:177-182.

Richardson C, Anderson M, Reid CL, Fox AM (2011) Neural indicators of error processing and intraindividual variability in reaction time in 7 and 9 year-olds. Dev Psychobiol 53:256-265.

Rueckert D, Sonoda LI, Hayes C, Hill DL, Leach MO, Hawkes DJ (1999) Nonrigid registration using free-form deformations: application to breast MR images. IEEE Trans Med Imaging 18:712-721.

Schmiedek F, Lövden M, Lindenberger U (2009) On the relation of mean reaction time and intraindividual reaction time variability. Psychol Aging 24:841-857.

Schneider W, Eschman A, Zuccolotto A (2002) E-prime user's guide. Pittsburgh: Psychological Software Tools Inc.

Smith SM (2002) Fast robust automated brain extraction. Hum Brain Mapp $17: 143-155$

Smith SM, Nichols TE (2009) Threshold-free cluster enhancement: addressing problems of smoothing, threshold dependence and localisation in cluster inference. Neuroimage 44:83-98.

Smith SM, Jenkinson M, Woolrich MW, Beckmann CF, Behrens TE, Johansen-Berg H, Bannister PR, De Luca M, Drobnjak I, Flitney DE, Niazy RK, Saunders J, Vickers J, Zhang Y, De Stefano N, Brady JM, Matthews PM (2004) Advances in functional and structural MR image analysis and implementation as FSL. Neuroimage 23 [Suppl 1]:S208-S219.

Smith SM, Jenkinson M, Johansen-Berg H, Rueckert D, Nichols TE, Mackay CE, Watkins KE, Ciccarelli O, Cader MZ, Matthews PM, Behrens TE (2006) Tract-based spatial statistics: voxelwise analysis of multi-subject diffusion data. Neuroimage 31:1487-1505.

Smith SM, Johansen-Berg H, Jenkinson M, Rueckert D, Nichols TE, Miller KL, Robson MD, Jones DK, Klein JC, Bartsch AJ, Behrens TE (2007) Acquisition and voxelwise analysis of multi-subject diffusion data with tract-based spatial statistics. Nat Protoc 2:499-503.

Stuss DT, Murphy KJ, Binns MA, Alexander MP (2003) Staying on the job: the frontal lobes control individual performance variability. Brain 126:2363-2380

Tamnes CK, Østby Y, Fjell AM, Westlye LT, Due-Tønnessen P, Walhovd KB (2010a) Brain maturation in adolescence and young adulthood: regional age-related changes in cortical thickness and white matter volume and microstructure. Cereb Cortex 20:534-548.

Tamnes CK, Østby Y, Walhovd KB, Westlye LT, Due-Tønnessen P, Fjell AM (2010b) Intellectual abilities and white matter microstructure in devel- 
opment: a diffusion tensor imaging study. Hum Brain Mapp 31:16091625.

Tsang JM, Dougherty RF, Deutsch GK, Wandell BA, Ben-Shachar M (2009) Frontoparietal white matter diffusion properties predict mental arithmetic skills in children. Proc Natl Acad Sci U S A 106:22546-22551.

Ullén F, Forsman L, Blom O, Karabanov A, Madison G (2008) Intelligence and variability in a simple timing task share neural substrates in the prefrontal white matter. J Neurosci 28:4238-4243.

Waber DP, De Moor C, Forbes PW, Almli CR, Botteron KN, Leonard G, Milovan D, Paus T, Rumsey J (2007) The NIH MRI study of normal brain development: performance of a population based sample of healthy children aged 6 to 18 years on a neuropsychological battery. J Int Neuropsychol Soc 13:729-746.

Wakana S, Jiang H, Nagae-Poetscher LM, van Zijl PC, Mori S (2004) Fiber tract-based atlas of human white matter anatomy. Radiology 230:77-87.

Walhovd KB, Fjell AM (2007) White matter volume predicts reaction time instability. Neuropsychologia 45:2277-2284.

Wechsler D (1999) Wechsler Abbreviated Scale of Intelligence (WASI). San Antonio, TX: The Psychological Corporation.

West R, Murphy KJ, Armilio ML, Craik FI, Stuss DT (2002) Lapses of inten- tion and performance variability reveal age-related increases in fluctuations of executive control. Brain Cogn 49:402-419.

Westlye LT, Walhovd KB, Bjørnerud A, Due-Tønnessen P, Fjell AM (2009) Error-related negativity is mediated by fractional anisotropy in the posterior cingulate gyrus-a study combining diffusion tensor imaging and electrophysiology in healthy adults. Cereb Cortex 19:293-304.

Westlye LT, Walhovd KB, Dale AM, Bjørnerud A, Due-Tønnessen P, Engvig A, Grydeland H, Tamnes CK, Østby Y, Fjell AM (2010) Life-span changes of the human brain white matter: diffusion tensor imaging (DTI) and volumetry. Cereb Cortex 20:2055-2068.

Williams BR, Hultsch DF, Strauss EH, Hunter MA, Tannock R (2005) Inconsistency in reaction time across the life span. Neuropsychology 19: $88-96$.

Williams BR, Strauss EH, Hultsch DF, Hunter MA (2007) Reaction time inconsistency in a spatial stroop task: age-related differences through childhood and adulthood. Aging Neuropsychol Cogn 14:417-439.

Woolrich MW, Jbabdi S, Patenaude B, Chappell M, Makni S, Behrens T, Beckmann C, Jenkinson M, Smith SM (2009) Bayesian analysis of neuroimaging data in FSL. Neuroimage 45:S173-186. 